\title{
State-specific reaction probabilities from a DVR-ABC Green function
}

\author{
Ward H. Thompson and William H. Miller \\ Department of Chemistry, University of California, Berkeley, CA 94720, USA \\ and Chemical Sciences Division, Lawrence Berkeley Laboratory, Berkeley, CA 94720. USA
}

Received 29 January 1993

\begin{abstract}
Seideman and Miller have recently introduced a direct, efficient method for calculating cumulative reaction probabilities by using a discrete variable representation to represent the Green function and absorbing boundary conditions to enforce the outgoing wave boundary conditions. It is shown that this method for representing the Green function can also be used to calculate statespecific reaction probabilities. Application to the collinear reaction $\mathrm{H}+\mathrm{H}_{2} \rightarrow \mathrm{H}_{2}+\mathrm{H}$ demonstrates that the method is an efficient way to calculate state-to-state and partially state-resolved reaction probabilities. In addition, distorted waves are useful in reducing the size of the DVR basis.
\end{abstract}

\section{Introduction}

In two recent papers [1-3] Seideman and Miller have shown that the cumulative reaction probability $N(E)$ for a chemical reaction, which is defined by

$N(E) \equiv \sum_{n_{\mathrm{r}}, n_{\mathrm{D}}}\left|S_{n_{\mathrm{p}, n_{\mathrm{r}}}}(E)\right|^{2}$,

can be computed directly and efficiently from the microcanonical reactive flux expression [4],

$N(E)=\frac{1}{2}(2 \pi h)^{2} \operatorname{tr}[\hat{F} \delta(E-\hat{H}) \hat{F} \delta(E-\hat{H})]$,

by using a discrete variable representation [5-7] (DVR) as the basis set in which to express the Hamiltonian and flux operators, $\hat{H}$ and $\hat{F}$, and absorbing boundary conditions [8] (ABC) to enforce the outgoing wave boundary conditions for the Green function from which the microcanonical density operator is obtained,

$\delta(E-H)=-\frac{1}{\pi} \operatorname{Im} \hat{G}^{+}(E)$.

$\left(n_{\mathrm{r}}\left(n_{\mathrm{p}}\right)\right.$ in eq. (1) denote quantum numbers of the reactants (products) and $\left\{S_{n_{\mathrm{p}, n_{\mathrm{f}}}}\right\}$ the reactive scattering $S$ matrix.) The canonical and microcanonical rate constants are given in terms of $N(E)$ by

$$
\begin{aligned}
& k(T)=\left[2 \pi h Q_{\mathrm{r}}(T)\right]^{-1} \\
& \times \int_{-\infty}^{\infty} \mathrm{d} E \exp (-E / k T) N(E),
\end{aligned}
$$

$k(E)=\left[2 \pi \hbar \rho_{\mathrm{r}}(E)\right]^{-1} N(E)$,

where $Q_{\mathrm{r}}$ and $\rho_{\mathrm{r}}$ are the reactant partition function and density of states, respectively. Other recent work closely related to this is that by Brown and Light [9] who have used a DVR with absorbing boundary conditions to evaluate thermal rate constants for collinear $\mathrm{H}+\mathrm{H}_{2}$ directly from the flux autocorrelation function.

It was also formally shown in the second paper [2] how this DVR-ABC Green function could be used to obtain state-to-state and partially state-resolved reaction probabilities. The purpose of the present Letter is to report that this proposal is indeed successful and to show some illustrative results. Within one general methodology - the DVR-ABC representation of the Green function - one thus has the capability of calculating fully state-to-state, partially state-resolved, or cumulative reaction probabilities. Section 2 first summarizes the theoretical methodology, and section 3 presents sample results for the 
workhorse test problem, collinear $\mathbf{H}+\mathrm{H}_{2}$.

\section{Summary of theory}

For concreteness, fig. 1 shows the contour plot of the potential energy surface [10] for the collinear $\mathrm{H}+\mathrm{H}_{2} \rightarrow \mathrm{H}_{2}+\mathrm{H}$ reaction with the DVR grid points for a typical state-to-state calculation indicated. (We use Colbert and Miller's [7] infinite order uniform DVR laid down in transition state normal coordinates.) Contours of an absorbing potential $\epsilon(q)$ are indicated by broken lines; $\epsilon(q)$ is zero in the interaction region and "turned on" in the reactant and product valleys. The grid point, or discrete variable matrix representation of the Green function is then

$\mathbf{G}(E)=(E \mid-H+\mathrm{i} \epsilon)^{-1}$,

where the "+" superscript on $\mathbf{G}$ is dropped. The absorbing potential may be thought of as a coordinatedependent version of the ubiquitous "i $e$ " that is added to the energy $E$ in formal scattering theory [11] to enforce outgoing wave boundary conditions

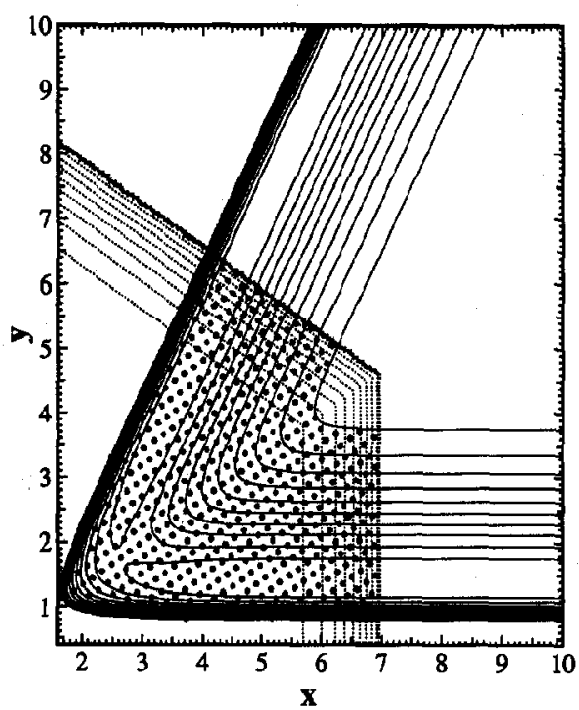

Fig. 1. Contour plot of the LSTH potential for collinear $\mathbf{H}+\mathrm{H}_{2}$; $x=R$ and $y=\sqrt{3} / 2 r$ are the mass-scaled reactant Jacobi coordinates. The dots represent the DVR grid points (laid down in the normal coordinates of the transition state) for a typical state-tostate calculation and the dashed contours show the quartic absorbing potential $\epsilon(q)$. for the Green function. The reader should see ref. [7] for the specific form of the DVR Hamiltonian matrix $\mathbf{H}$ and ref. [1] for a more complete description of the DVR-ABC Green function. Refs. [1-3] then showed that eq. (1b) for the cumulative reaction probability takes the very simple form

$N(E)=4 \operatorname{tr}\left[\mathbf{G}(E)^{*} \cdot \epsilon_{\mathrm{p}} \cdot \mathbf{G}(E) \cdot \epsilon_{\mathrm{r}}\right]$,

where $\varepsilon_{\mathrm{r}}\left(\epsilon_{\mathrm{p}}\right)$ is the part of $\epsilon$ in the reactant (product) absorbing region.

The state-to-state version of the theory starts with a formally exact expression for an $S$-matrix element (for a reactive or non-reactive process),

$$
\begin{gathered}
S_{n_{\mathrm{p}}, n_{\mathrm{r}}}(E)=S_{n_{\mathrm{p}}, n_{\mathrm{r}}}^{0}(E)+\frac{\mathrm{i}}{\hbar}\left[\left\langle\Phi_{n_{\mathrm{p}}}|\hat{H}-E| \Phi_{n_{\mathrm{r}}}\right\rangle\right. \\
\left.+\left\langle(\hat{H}-E) \Phi_{n_{\mathrm{p}}}\left|\hat{G}^{+}(E)\right|(\hat{H}-E) \Phi_{n_{\mathrm{r}}}\right\rangle\right],
\end{gathered}
$$

where $\Phi_{n}$ is any (regular) scattering wavefunction with a unit incoming wave in channel $n$ and outgoing waves in all other open channels $n^{\prime}$

$$
\begin{gathered}
\Phi_{n}(\boldsymbol{r}, R) \sim-\frac{\exp \left(-\mathrm{i} k_{n} R\right)}{v_{n}^{1 / 2}} \phi_{n}(\boldsymbol{r}) \\
+\sum_{n^{\prime}} \frac{\exp \left(\mathrm{i} k_{n^{\prime}} R\right)}{v_{n^{\prime}}^{1 / 2}} \phi_{n^{\prime}}(\boldsymbol{r}) S_{n^{\prime}, n}^{0} .
\end{gathered}
$$

( $r$ in eq. (7) denotes all coordinates of the system other than that for relative translation, $R$, and $\left\{\phi_{n}(r)\right\}$ the channel eigenfunctions; $v_{n}$ is the translational velocity for channel $n$.) The simplest choice for $\Phi_{n}$, and the one used for the results described in section 3 , is

$\Phi_{n}(\boldsymbol{r}, R)=-\frac{\exp \left(-\mathrm{i} k_{n} R\right)}{v_{n}^{1 / 2}} \phi_{n}(\boldsymbol{r})$,

for which

$S_{n^{\prime}, n}^{0}=0$.

A more conventional choice would be the standing free wave

$$
\begin{aligned}
& \Phi_{n}(r, R)=-2 \mathrm{i} \frac{\sin k_{n} R}{v_{n}^{1 / 2}} \phi_{n}(r) \\
& =\left(-\frac{\exp \left(-\mathrm{i} k_{n} R\right)}{v_{n}^{1 / 2}}+\frac{\exp \left(\mathrm{i} k_{n} R\right)}{v_{n}^{1 / 2}}\right) \phi_{n}(\boldsymbol{r}),
\end{aligned}
$$

for which

$S_{n^{\prime}, n}^{0}=\delta_{n^{\prime}, n}$. 
Both choices work, but we find that the incoming wave in eqs. (8) leads to much more rapid convergence.

In calculating partially state-resolved reaction probabilities we have also used distorted waves [12] for $\Phi_{n}$, which is useful in reducing the size of the grid needed to represent the Green function because the absorbing potential can be brought in closer to the reactive region. (Non-reactive distorted waves have also been used analogously in $S$-matrix Kohn variational calculations of reactive scattering [13].) $\Phi_{n}$ then has the form

$\Phi_{n}(r, R)=\sum_{n^{\prime}} \phi_{n^{\prime}}(r) u_{n^{\prime} \leftarrow n}(R)$,

where the radial function matrix $\left\{u_{n^{\prime}-n}(R)\right\}$ is taken to have the asymptotic form

$u_{n^{\prime} \leftarrow n}(R) \sim \delta_{n^{\prime}+n} \frac{\exp \left(-\mathrm{i} k_{n} R\right)}{v_{n}^{1 / 2}}$

for large $R$ (therefore $S_{n^{\prime}, n}^{0}=0$ ), and the inelastic scattering coupled-channel equations are integrated inward to obtain it for all $R$. We have used the standard Numerov algorithm for work in this Letter, though any inelastic propagation algorithm would suffice. We note that for the free or distorted incoming radial function it is not necessary to multiply it by a cutoff function to regularize it for small $R$ (as is necessary in the Kohn variational approach [7]); this is because $\Phi_{n}$ is always multiplied by the reactant or product absorbing potential (cf. eqs. (12) and (15) below) which vanishes identically for small $R$.

The DVR-ABC Green function is now used in eq. (6), and by making use of the matrix identity

$(\mathbf{H}-E \mathbf{I}) \cdot \mathbf{G}(E)=-\mathbf{I}+\mathrm{i \epsilon} \cdot \mathbf{G}(E)$,

one readily obtains

$S_{n_{\mathrm{p}}, n_{\mathrm{r}}}(E)=-\frac{i}{\hbar} \boldsymbol{\Phi}_{n_{\mathrm{p}}}^{\mathrm{T}} \cdot \boldsymbol{\epsilon}_{\mathrm{p}} \cdot \mathbf{G}(E) \cdot \boldsymbol{\epsilon}_{\mathrm{r}} \cdot \boldsymbol{\Phi}_{n_{\mathrm{r}}}$,

where $\boldsymbol{\Phi}_{n}$ is the following DVR vector in grid point space

$\left(\boldsymbol{\Phi}_{n}\right)_{j}=w_{j}^{1 / 2} \Phi_{n}\left(\boldsymbol{q}_{j}\right)$,

where $q$ denotes all coordinates, and $q_{j}$ and $w_{j}$ are the (multidimensional) grid points and DVR weights, respectively.

Finally, using similar formal relations one is able to obtain an intcrmcdiatc lcvel of state resolution between the complete state-to-state result, eq. (12), and the cumulative reaction probability, eq. (5). Specifically, the total reaction probability for a given initial reactant state,

$P_{n_{t}}(E) \equiv \sum_{n_{\mathrm{p}}}\left|S_{n_{\mathrm{p}, n_{\mathrm{r}}}}(E)\right|^{2}$,

is given by

$P_{n_{\mathrm{r}}}(E)=\frac{2}{\hbar} \boldsymbol{\Phi}_{n_{\mathrm{r}}}^{\mathrm{T} *} \cdot \boldsymbol{\epsilon}_{\mathrm{r}} \cdot \mathbf{G}^{*}(E) \cdot \boldsymbol{\epsilon}_{\mathrm{p}} \cdot \mathbf{G}(E) \cdot \boldsymbol{\epsilon}_{\mathrm{r}} \cdot \boldsymbol{\Phi}_{n_{\mathrm{r}}} \cdot$

As noted before [3], all three of these expressions, eqs. (5), (12) and (15), are consistent if the following completeness identity holds:

$(2 \hbar)^{-1} \sum_{n_{\mathrm{r}}} \epsilon_{\mathrm{r}} \cdot \Phi_{n_{\mathrm{r}}} \Phi_{n_{\mathrm{r}}}^{\mathrm{T} *} \cdot \epsilon_{\mathrm{r}}=\epsilon_{\mathrm{r}}$

and similarly for the product arrangement; the Appendix derives this identity for the DVR-ABC space.

\section{Results}

To test the state-resolved aspects of this DVR-ABC methodology, we have computed state-to-state and partially state-resolved reaction probabilities for the collinear $\mathrm{H}+\mathrm{H}_{2}$ reaction on the LSTH potential [10].

\subsection{State-to-state reaction probability}

In computing the state-to-state reaction probabilities from eq. (12) the DVR grid is laid down in the normal mode coordinates of the transition state $\left(q_{1}\right.$, $q_{2}$ ) (just as in ref. [5]) and truncated by an energy cutoff, i.e. if the potential at a given DVR point is greater than some value $V_{\text {cut }}$, then that point is discarded. The grid is also truncated by the boundaries of the absorbing potential. The absorbing potentials in the reactant and product regions, $\epsilon_{\mathrm{r}}$ and $\epsilon_{\mathrm{p}}$, are taken to be a function of the translational Jacobi coordinate for each arrangement; i.e.

$\epsilon_{y}\left(q_{1}, q_{2}\right)=\epsilon\left(R_{\gamma}\left(q_{1}, q_{2}\right)\right)$

for $\gamma=\mathrm{r}$ (reactants) or $\mathrm{p}$ (products), where $R_{\mathrm{r}}$ and $R_{\mathrm{p}}$ are functions of the transition state normal coordinates $\left(q_{1}, q_{2}\right)$. For all calculations in this Letter, 
the absorbing potential has been taken to be a quartic function, beginning at $R_{0}$ and ending at $R_{\max }$,

$\epsilon_{\gamma}\left(R_{\gamma}\right)=\lambda\left(\frac{R_{\gamma}-R_{0}}{R_{\max }-R_{0}}\right)^{4}$,

though we note that other functional forms work essentially as well. The density of the DVR grid points is specified by the grid constant, $N_{\mathrm{B}}$, which is the number of points per de Broglie wavelength. This is related to the spacing between points, $\Delta q$, by $N_{\mathrm{B}}=2 \pi$ / $k \Delta q$ where $k=\left(2 m E / \hbar^{2}\right)^{1 / 2}$.

There are essentially three convergence parameters to consider in these calculations: (1) $N_{\mathrm{B}}$ must be large enough to properly represent the Green function on the DVR grid; (2) $V_{\text {cut }}$ must be large enough to include all physically relevant regions of the potential energy surface; and (3) $R_{\max }$ must be large enough for the absorbing potential to absorb all the outgoing flux without reflection.

Fig. 2 illustrates the convergence of the reactive state-to-state probabilities at a total energy of $1.0 \mathrm{eV}$ as the size of the grid is increased, for different values of $V_{\text {cut }}$. (The percent error is calculated by comparison to the results of Bondi and Connor [14].) At this energy there are two open vibrational channels. The minimum grid size for which all the reaction probabilities are converged to within $1 \%$ error has $R_{\max }=6.9$ au with $N_{\mathrm{B}}=3.7$ and $V_{\text {cut }}=4.1 \mathrm{eV}$, corresponding to 546 DVR grid points. Agreement with the exact answer to within $3 \%$ can be obtained with $R_{\max }=6.7 \mathrm{au}, V_{\text {cut }}=3.0 \mathrm{eV}$ and $421 \mathrm{DVR}$ points. Similar convergence behavior can be seen for the grid constant and the cutoff energy.

\subsection{Total reaction probability for a given initial state}

For computing the total reaction probability for a given initial reactant state (via eq. (15)), the DVR grid was again laid down in the normal mode coordinates of the transition state (cf. fig. 3 ). The absorbing potentials $\epsilon_{\mathrm{r}}$ and $\epsilon_{\mathrm{p}}$ were chosen as above, eqs. (17) and (18), though now the parameters $R_{0}$ and $R_{\max }$ are different for the reactant and product absorbing potentials. I.e. since no state-specific information is being obtained about the products, the product absorbing potential can be brought in much
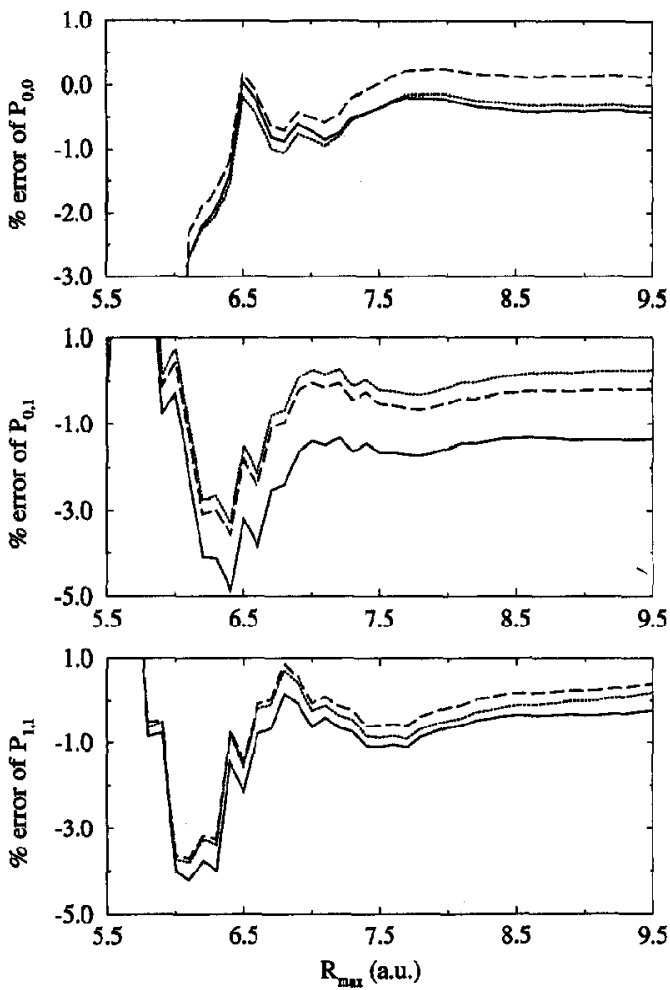

Fig. 2. Percent error of the state-to-state reaction probabilities as a function of $R_{\max }$, with $N_{\mathrm{B}}=3.7$, for different values of the cutoff energy: (-) $V_{\text {cut }}=3.0 \mathrm{eV}$; $(--) V_{\text {cur }}=4.1 \mathrm{eV}$; and (- - ) $V_{\text {cut }}=5.0 \mathrm{eV}$.

closer to the transition state - very much in the spirit of the cumulative reaction probability calculation while the reactant absorbing potential remains essentially the same as in the state-to-state case.

Fig. 4 shows the convergence for $E=1.0 \mathrm{eV}$ with respect to $R_{\mathrm{r}, \max }$ for different values of $V_{\text {cut }}$. For comparison, the minimum grid needed to obtain $<1 \%$ error has $R_{\mathrm{r}, \max }=6.8 \mathrm{au}, R_{\mathrm{p}, \max }=5.2 \mathrm{au}, N_{\mathrm{B}}=3.2$, and $V_{\text {cut }}=3.8 \mathrm{eV}$ corresponding to 290 DVR points. However, the reaction probabilities converge to within $3 \%$ for $V_{\text {cut }}=3.1 \mathrm{eV}$ and a grid as small as 231 points.

Finally, when state-resolved information is being obtained - either state selection for both reactants and products (eq. (12)), or only for reactants (eq. (15)) - the absorbing potential can be brought in closer, and the size of the DVR grid thus reduced, by using distorted waves for the incoming wavefunction $\Phi_{n}$. Fig. 5 shows the initial state-selected reaction 


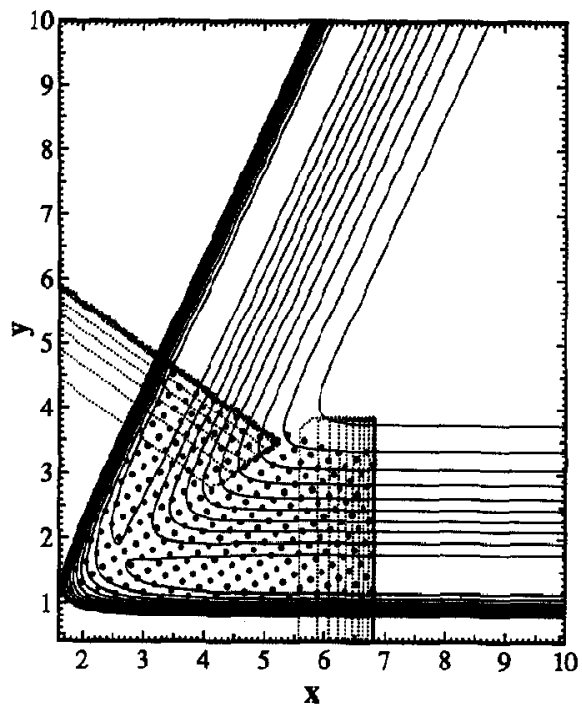

Fig. 3. Same as in fig. 1 with a grid and absorbing potential typical for a calculation of the total reaction probability from a given initial reactant state.

probabilities over a range of total energies for two different grid sizes compared to the results of Bondi and Connor. For the probability of reaction from the ground vibrational state, $P_{0}(E)$, the agreement is excellent for both values of $R_{\mathrm{r} \text {, max }}$, except near the resonance at $0.9 \mathrm{eV}$ where the error is on the order of several percent. For $P_{1}(E)$ reasonable agreement is obtained with the smaller grid while for the larger grid the agreement is within 1\% except around 0.9 $\mathrm{eV}$. The difficulty around this energy is due to the low translational energies associated with the excited vibrational state. These low translational energies require a very slow turn on of the absorbing potential to avoid reflection. It should be noted that the parameters describing the grid and absorbing potential were obtained by converging the reaction probabilities at $E=1.0 \mathrm{eV}$. It is encouraging that such agreement can be obtained over the entire energy range using one set of parameters. For comparison with the above results, the minimum grid size needed to converge to within $1 \%$ at $E=1.0 \mathrm{eV}$ is $216 \mathrm{DVR}$ points.

\section{Concluding remarks}

These results thus show that the DVR-ABC Green
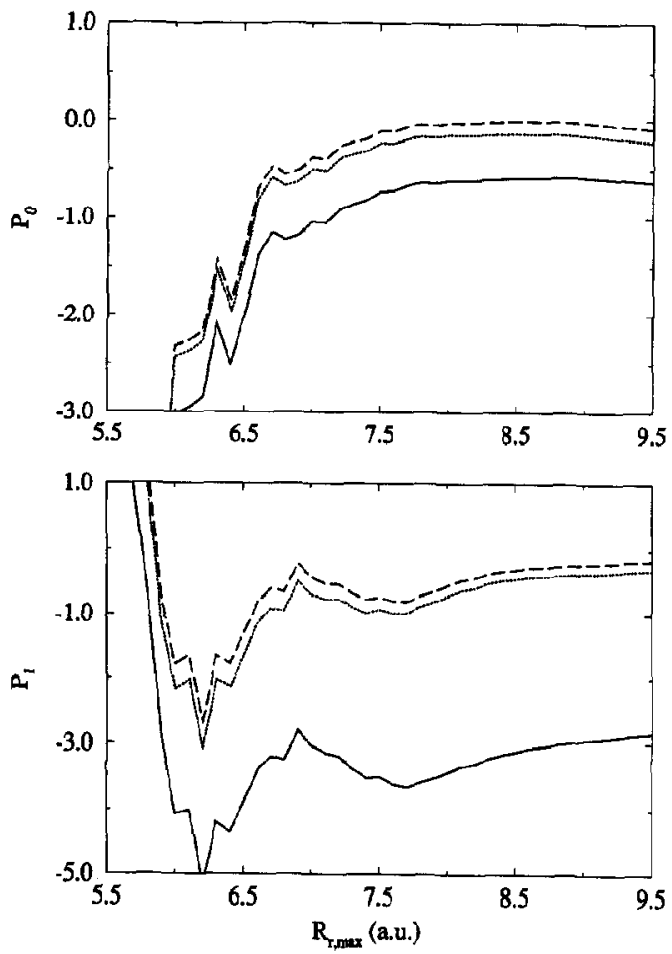

Fig. 4. Percent error of the total reaction probability from a given initial state probability as a function of $R_{r, \max }$, with $N_{\mathrm{B}}=3.2$ and $R_{p, \max }=5.2$ au, for different values of $V_{\text {eut }}:(\longrightarrow) V_{\text {eut }}=3.0 \mathrm{eV}$; $(--) V_{\text {cul }}=3.8 \mathrm{eV}$; and $(---) V_{\text {cut }}=4.5 \mathrm{eV}$.

function provides an efficient method for calculating state-to-state and partially state-resolved reaction probabilities (as well as the cumulative reaction probability). In addition, for the partially state-resolved version, we have seen that distorted waves can be used to significantly reduce the size of the DVR basis. It should be noted that in computing the $S$-matrix elements via eq. (12), or the initial state-selected probabilities by eq. (15), one has to solve the set of linear equations

$(E \mathrm{I}-\mathrm{H}+\mathrm{i} \epsilon) \cdot \boldsymbol{x}=\epsilon_{\mathrm{T}} \cdot \boldsymbol{\Phi}_{n_{\mathrm{r}}}$,

to obtain

$\boldsymbol{x} \equiv \boldsymbol{G} \cdot \boldsymbol{\epsilon}_{\mathbf{r}} \cdot \boldsymbol{\Phi}_{n_{\mathbf{r}}}$

only once for the initial state of interest. This is a distinct advantage over time-independent variational methods where there is no significant savings in cal- 

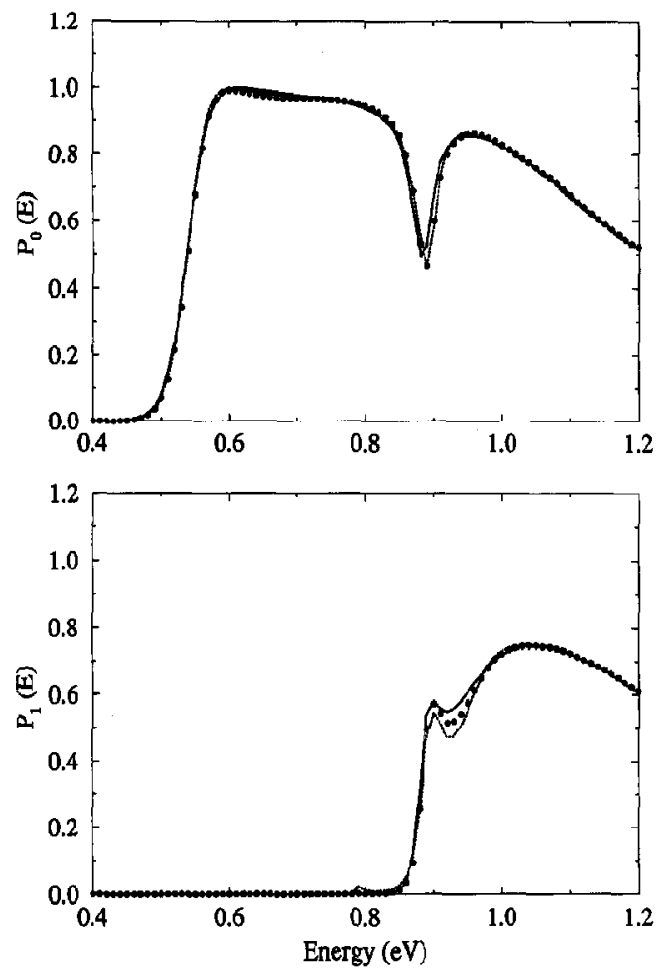

Fig. 5. Total reaction probability from a given initial state as a function of energy computed using distorted waves for (-.) $R_{\mathrm{r}, \max }=5.5$ au and $(O) R_{\mathrm{r}, \max }=6.5$ au compared to the exact results of (—) Bondi and Connor (ref. [10]).

culating one column of the $S$ matrix rather than the entire $S$ matrix.

\section{Acknowledgement}

This work was supported by the Director, Office of Energy Research, Office of Basic Energy Sciences, Chemical Sciences Division of the US Department of Energy under Contract No. DE-AC03-76SF00098. WHT acknowledges support from the National Science Foundation.

\section{Appendix. A closure identity for the DVR-ABC Green function}

Eq. (16) at first looks nonsensical because the left- and right-hand sides contain different factors of the absorbing potential $\epsilon_{\mathrm{r}}\left(\Phi_{n_{\mathrm{r}}}\right.$ is independent of $\left.\epsilon_{\mathrm{r}}\right)$. Indeed, it is only valid if the DVR-ABC Green function multiplies it from the left and the right, respectively, as they do in eqs. (5), (12), and (15). The following analysis shows how this closure relation, eq. (16), comes about.

One begins with the two operator identities

$$
\begin{aligned}
& \delta(E-\hat{H})=-\frac{1}{\pi} \operatorname{Im} \hat{G}^{+}(E), \\
& \delta(E-\hat{H})=\sum_{n} \rho\left|\Psi_{E, n}\right\rangle\left\langle\Psi_{E, n}\right|,
\end{aligned}
$$

where $\Psi_{E, n}$ is the exact scattering wavefunction with boundary conditions of the form of eq. (7) (but where in this case $S^{0}$ is the exact $S$ matrix, $S$; the density of states factor $\rho=(2 \pi \hbar)^{-1}$ for the normalization of the translational wavefunctions used in eq. (7)). Working first on eq. (A.2), a formal expression for $\Psi_{E, n}$ in terms of the zeroth-order scattering wavefunction $\Phi_{n}$ is

$$
\begin{aligned}
\Psi_{E, n} & =\left[1+\hat{G}^{+}(E)(\hat{H}-E)\right] \Phi_{n} \\
= & \hat{G}^{+}(E)(E-\hat{H}+\mathrm{i} \hat{\epsilon}+\hat{H}-E) \Phi_{n} \\
= & \hat{G}^{+}(E) \mathbf{i} \hat{\epsilon} \Phi_{n}
\end{aligned}
$$

a well-known result for $\epsilon$ a positive constant, but we note here that it also applies in our more general case that $\hat{\epsilon}$ is an operator. Using (A.3) in eq. (A.2), and putting this into a DVR thus gives

$\delta(E \mathbf{I}-\mathbf{H})=(2 \pi h)^{-1} \sum_{n} \mathbf{G} \cdot \boldsymbol{\epsilon} \cdot \boldsymbol{\Phi}_{n} \boldsymbol{\Phi}_{n}^{\mathrm{T} *} \cdot \boldsymbol{\epsilon} \cdot \mathbf{G}^{*}$,

where the "+" designation has been dropped from G.

Turning now to eq. (A.1), one has

$\operatorname{Im} \hat{G}^{+}$

$$
\begin{aligned}
& =\operatorname{Im}(E+\mathrm{i} \hat{\epsilon}-\hat{H})^{-1}(E-\mathrm{i} \hat{\epsilon}-\hat{H})(E-\mathrm{i} \hat{\epsilon}-\hat{H})^{-1} \\
& =-\hat{G}^{+} \hat{\epsilon} \hat{G}^{+*},
\end{aligned}
$$

the DVR of which, used in eq. (A.1), gives

$$
\delta(E \mathbf{I}-\mathbf{H})=\frac{1}{\pi} \mathbf{G} \cdot \mathbf{\epsilon} \cdot \mathbf{G}^{*} .
$$


Equating eqs. (A.4) and (A.6) is seen to lead to the relation in eq. (16).

\section{References}

[1] T. Seideman and W.H. Miller, J. Chem. Phys. 96 (1992) 4412.

[2] T. Seideman and W.H. Miller, J. Chem. Phys. 97 (1992) 2499.

[3] W.H. Miller and T. Seideman, in: Time dependent quantum molecular dynamics: experiment and theory, ed. J. Broeckhove, NATO ARW (1992).

[4] W.H. Miller, S.D. Schwartz and J.W. Tromp, J. Chem. Phys. 79 (1983) 4889

[5] D.O. Harris, G.G. Engerholm and W.D. Gwinn, J. Chem. Phys. 43 (1965) 1515.

[6] J.V. Lill, G.A. Parker and J.C. Light, Chem. Phys. Letters 89 (1982) 483;

J.C. Light, I.P. Hamilton and J.V. Lill, J. Chem. Phys. 82 (1985) 1400;

J.C. Light, R.M. Whitnell, T.J. Park and S.E. Choi, NATO ASI Ser. 277 (1989) 187

[7] D.T. Colbert and W.H. Miller, J. Chem. Phys. 96 (1992) 1982.
[8] A. Goldberg and B.W. Shore, J. Phys. B 11 (1978) 3339; C. Leforestier and R.E. Wyatt, J. Chem. Phys. 78 (1983) 2334;

C. Cerjan, D. Kosloff and T. Teshef, Geophysics 50 (1985) 705 ;

R. Kosloff and D. Kosloff, J. Comput. Phys. 63 (1986) 363;

D. Neuhauser and M. Baer, J. Chem. Phys. 90 (1989) 4351;

D. Neuhauser, M. Baer and D.J. Kouri, J. Chem. Phys. 93 (1990) 2499;

D. Neuhauser, J. Chem. Phys. 93 (1990) 7836.

[9] D. Brown and J.C. Light, J. Chem. Phys. 97 (1992) 5465.

[10] P. Siegbahn and B. Liu, J. Chem. Phys. 68 (1978) 2457; D.G. Truhlar and C.J. Horowitz, J. Chem. Phys. 68 (1978) 2566; 71 (1979) 1514.

[11] R.G. Newton, Scattering theory of waves and particles, 2nd Ed. (Springer, Berlin, 1982) pp. 176ff.

[12] L.M. Hubbard, S. Shi and W.H. Miller, J. Chem. Phys. 78 (1983) 2381;

B.R. Johnson, Chem. Phys. Letters 13 (1972) 172.

[13] J.Z.H. Zhang and W.H. Miller, J. Chem. Phys. 91 (1989) 1528 ;

J.Z.H. Zhang, D.L. Yeager and W.H. Miller, Chem. Phys. Letters 173 (1990) 489;

G.C. Groenenboom, D.T. Colbert and W.H. Miller, manuscript in preparation.

[14] D.K. Bondi and J.N.L. Connor, J. Chem. Phys. 82 (1985) 4383. 